\section{Industry advances landmark CRISPR candidate into the clinic}

Vertex Pharmaceuticals and partner CRISPR Therapeutics have become the first US and European companies to move a CRISPR-based gene-editing product into the clinic, launching a trial of CTX001 in patients with $\beta$-thalassaemia.

Researchers first reported that CRISPR proteins could cut DNA in human cells in 2013, quickly raising hopes that these proteins might be harnessed as a new gene-editing modality. In the case of Vertex and CRISPR Therapeutics' CTX001 therapy, the technology is used to cut the DNA that encodes BCL11A, a transcription factor that typically represses production of fetal haemoglobin. In patients with $\beta$-thalassaemia, who carry mutations in the $\beta$-globin gene that compromise production of normal haemoglobin, CRISPR-induced disruption of BCL11A enables expression of compensatory fetal haemoglobin.

CTX001 is an autologous ex vivo therapy, in which haematopoietic stem and progenitor cells are harvested from patients, edited and then re-infused back into the patients.

This type of strategy is typically considered to be less scientifically challenging than in vivo gene editing, because it side-steps drug delivery hurdles and reduces the risk of off-tissue editing.

The phase I/II trial will enroll 12 subjects in Europe. The FDA put the trial on clinical hold in the US before it started, "pending the resolution of certain questions".

Other companies are also quickly advancing CRISPR products towards the clinic. Editas Medicine and Allergan plan to file later this year for approval to test EDIT-101, a CRISPR product that cuts out a mutation in CEP290, as an in vivo treatment for Leber congenital amaurosis. Intellia Therapeutics has partnered with Regeneron on an in vivo CRISPR product for the treatment of transthyretin amyloidosis, and with Novartis on an ex vivo product for sickle cell disease.

Sangamo Therapeutics, another gene-editing pioneer, has meanwhile advanced a few zinc-finger nuclease (ZFN)-based therapies into the clinic. In September it reported mixed data with its first in vivo gene-editing therapy. SB-913 places a normal functioning copy of the gene coding for iduronate 2-sulfatase (IDS) - under the control of the strong albumin promoter in the liver - in patients with the lysosomal storage disorder Hunter syndrome, which is caused by a lack of functional IDS. Treatment with SB-913 reduced disease-associated urinary glycosaminoglycan levels in two patients, but the company was unable to detect increased plasma levels of IDS. In March, the company also launched a phase I/II collaboration with Bioverativ, a Sanofi company, to test ST-400, a ZFN-based editor of BCL11A for the treatment of $\beta$-thalassaemia.

Asher Mullard

\section{Chi-Med scores key approval for 'discovered in China' cancer drug}

Chinese drug regulators approved Chi-Med's VEGFR inhibitor fruquintinib for the treatment of metastatic colorectal cancer, providing the first unconditional approval for a homegrown new drug in China that has been tested in randomized clinical trials.

Although industry has long been ramping up its investments in China, many of the early ventures were focused on outsourcing chemistry and manufacturing services. Some firms have been building end-to-end drug discovery capabilities in China as well (Nat. Rev. Drug Discov. 16, 443-446; 2017). Chi-Med, one such company, has now scored a landmark approval that they hope will prove their ability to discover and develop follow-on drug contenders with best-in-class potential.

First-generation VEGFR inhibitors like Bayer and Amgen's sorafenib are multikinase inhibitors with broad binding profiles and toxicity liabilities. Chi-Med by contrast designed its anti-angiogenesis agent fruquintinib to act as a hyperselective VEGFR inhibitor, with the aims of minimizing off-target toxicities, improving tolerability and providing more consistent target coverage. For now, the drug is only approved in China for patients who have failed at least two prior lines of therapy, and the company has yet to prove its best-in-class status. But further clinical trials are underway, including a phase I trial in the US.

A few China-based R\&D teams are also pursuing potential first-in-class,
discovered-in-China drugs. Novartis's anticancer drug MAK683, an EED inhibitor that was discovered in China, is in a phase I/II trial that is set to wrap up next year. Roche's capsid inhibitor RO7049389 (also known as RG7907) and its TLR7 agonist RO7020531 (RG7854) are in phase I trials for hepatitis B virus infection.

Asher Mullard

\section{Painkilling anti-NGF antibodies stage phase III comeback}

Regulators placed late-stage anti-NGF antibodies on clinical hold nearly 8 years ago, over concerns that these novel pain-killing drugs were causing joint destruction and autonomic dysfunction. After years of slow-going progress, two of these non-opioid drugs are now generating promising phase III data again.

In July, Pfizer and partner Eli Lilly reported that tanezumab hit the primary end points change in pain, function and global assessment at 16 weeks - in a phase III trial involving 698 patients with osteoarthritis. The trial didn't lead to any cases of osteonecrosis, the red flag that first triggered the safety review of these drugs. Rapidly progressive osteoarthritis was observed in tanezumab-treated patients at a frequency of less than $1.5 \%$, and was not observed in the placebo arm.

Phase III trials of the drug are ongoing in chronic low back pain and in cancer pain, with results expected by the end of the year. The companies are on track to file for regulatory approval of the antibody in 2019.

In August, Regeneron and partner Teva reported that their phase III fasinumab hit co-primary end points - change in pain and change in function at 16 weeks — in a 646-patient osteoarthritis trial. No cases of osteonecrosis have been identified to date in this study. Among the approximately $65 \%$ of patients who had completed a first radiographic assessment, the placebo-adjusted rate of adjudicated joint damage was approximately $2 \%$. In May, however, an independent data monitoring committee recommended that high-dose regimens of fasinumab be discontinued based on the risk-benefit assessment.

A phase III trial of fasinumab in chronic low back pain is ongoing.

Other companies including Amgen, AstraZeneca, AbbVie and Johnson \& Johnson have discontinued development of their anti-NGF antibodies.

Asher Mullard 